Nuit blanche

\title{
Ainsi parlait Zarathoustra
}

\section{Jean Désy}

Numéro 90, printemps 2003

URI : https://id.erudit.org/iderudit/19200ac

Aller au sommaire du numéro

Éditeur(s)

Nuit blanche, le magazine du livre

ISSN

0823-2490 (imprimé)

1923-3191 (numérique)

Découvrir la revue

Citer ce compte rendu

Désy, J. (2003). Compte rendu de [Ainsi parlait Zarathoustra]. Nuit blanche, (90), 20-21.

Ce document est protégé par la loi sur le droit d'auteur. L'utilisation des services d'Érudit (y compris la reproduction) est assujettie à sa politique d'utilisation que vous pouvez consulter en ligne.

https://apropos.erudit.org/fr/usagers/politique-dutilisation/
Cet article est diffusé et préservé par Érudit.

Érudit est un consortium interuniversitaire sans but lucratif composé de l’Université de Montréal, l’Université Laval et l'Université du Québec à Montréal. Il a pour mission la promotion et la valorisation de la recherche. https://www.erudit.org/fr/ 


\section{Ainsi parlait Zarathoustra}

Par

Jean Désy*

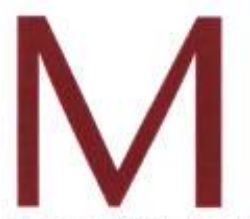

on " livre jamais lu » m'est tombé des mains. J'avais commencé à le lire. Il est demeuré dans la case des « jamais lus ». Ce n'est pas que je n'avais pas envie de le parcourir. J'avais déjà abordé une grande partie de l'œuvre de son auteur. Mais ce livre, sans doute le plus terrible de ses écrits, le plus incisif en tout cas, je n'ai pu le terminer. Je n'ai pu aller très loin. Je n'ai tourné qu'une centaine de pages. Pourquoi ? Parce que j'avais été pris de nausée. Mon « livre jamais lu " m’a dérangé.

Il m’a bouleversé. Que peut-on demander de mieux à un livre que de nous mordre, de nous piquer, de nous émouvoir, de nous transformer? Il n'y a probablement que ce type de livre qui laisse sa marque dans l'esprit, mais le problème, c'est que le livre qui nous perturbe l'âme se trouve aux antipodes de ce qui, jusque-là, avait formé notre vision du monde. Tout livre perturbateur est une révélation, mais toute révélation se fait dans le déséquilibre, laissant apparaitre la part d'ombre, la part sombre et épouvantée qui habite en nous.

Mon « livre jamais lu ", je l'ai donc repoussé. Je l'ai rangé dans ma bibliothèque parmi les autres du même auteur. À maintes reprises, je l'ai retiré de son étagère, je l'ai placé tout près de mon pupitre, je l'ai apporté avec moi en voyage. Jean Désy Mais, chaque fois, il s'est passé la même
chose. Ce livre m'avait ébranlé. La même nausée m'a envahi. J'ai dû le laisser, encore et encore. C'est ainsi. Je peux maintenant affirmer qu'il s'agit du livre le plus difficile sur lequel je me suis acharné au cours de ma vie, le plus essentiel, probablement, ou l'un des plus essentiels en tout cas.

Certains livres m'ont réconforté, ils m'ont appris beaucoup sur moi-même et sur le monde, tandis que ce livre " jamais lu ", lui, n'a cessé de me replonger dans les fondements mêmes de mon angoisse existentielle. C'est probablement l'une des raisons de la nausée qu'il fait naître en moi. Il y a son ton et son style, extrêmement efficaces, qui contribuent à me le rendre indigeste.
Il y a aussi l'extraordinaire façon avec laquelle son auteur emmêle intelligence logique et irrationnelle intuition. Dans ce livre, il existe un mélange subtil, tout à fait dramatique, de rationalité et d'irrationalité. Les phrases sont ordonnées, la syntaxe respectée, il n'existe pas vraiment de jeu formel entre les mots. Ce sont plutôt les idées, les avancées dans le monde de la pensée, qui se présentent sur un mode qu'on pourrait qualifier de " poétique ". Ce livre n'est pourtant pas un recueil de poèmes. Pas un roman non plus. Il n'a pas été pensé en vers. Son ton le rapproche du cri, de l'invective, du sermon. Dans ce livre, on hurle des choses qui, d'ailleurs, ne seraient que murmurées. J'ai bu l'lliade et l'Odyssée. J'ai dévoré Don Quichotte. Je me suis rassasié de Hamlet et de Cent ans de solitude. Mais mon livre jamais lu, il m'a désarçonné, il m’a fait tomber de ma chaise. Ce livre, c'est : Ainsi parlait Zarathoustra, de Friedrich Nietzsche.

Il est question d'âme. C'est ce qui le rend si important, mais si intolérable aussi. André Malraux a dit que le XXIe siècle serait celui de la spiritualité, ou quil ne serait pas. Dans Ainsi parlait Zarathoustra, Nietzsche formule la mort de Dieu. Le philosophe n'a pas tué Dieu. Il n'a fait que constater cette mort. Au XVIII' siècle, en Occident, Dieu agonisait.

Nietzsche, qui l'avait compris, a été le plus formidable porte-parole de ce qui s'est passé dans l'Histoire et de ce qui allait se passer. Sa parole a retenti. Elle a essaimé. Et cette mort de Dieu, présentée, annoncée, justifiée et attestée, elle me trouble.

Aucun écrivain contemporain ne me semble avoir dépassé Nietzsche. Aucun penseur, aucun poète n'est allé aussi loin dans la représentation de ce que serait le $\mathrm{XX}^{\mathrm{e}}$ siècle, mais aussi le XXI" siècle. Il y a là vision prémonitoire. Encore aujourd'hui, je relis Le gai savoir ou La naissance de la tragédie pour savoir où j'en suis, pour savoir où nous en sommes dans cet immense capharnaüm qu'est le monde actuel, pour comprendre un peu mieux ce qui s'est passé, mais aussi ce qui va se passer, pour intervenir peut-être - mais quelles prétentions pourrais-je bien avoir, moi, le pou, la puce, le lemming ? - pour apprendre à me protéger, non pas pour sauver ma peau, mais pour sauver mon âme.

Pendant longtemps, j'ai cru qu'Ainsi parlait 
Zarathoustra remettait ma foi en question. Puis, j'ai compris que le mot " Dieu " pouvait être remplacé par " transcendance ». C'est la mort de la transcendance, de l'aspect transcendant de toute l'activité humaine, que Nietzsche a proclamé de façon si catégorique. La moitié du XX $\mathrm{XX}^{\mathrm{e}}$ siècle a été vécue par la plupart des groupes humains dans une totale absence de transcendance. Il le fallait. L'humanité avait suffisamment dénaturé les figures de Dieu et du transcendant. Il fallait qu'elles meurent. Et Dieu est mort. Or, quiconque est possédé par l'irrationnelle certitude de la vie au-delà de la vie, de la mort amalgamée à la vie, ne peut qu'être ébranlé par l'annonce de la mort de Dieu. C'est alors que la lecture du « livre jamais lu " fait à nouveau partie du possible, de l'inéluctable. C'est alors qu'il est temps de replonger dans la découverte du surhumain, de cet être qui a les deux pieds sur terre, solides, mais qui se trouve pieds nus dans la mousse humide. Regarde-t-il vers le ciel, ce surhumain? Prie-t-il pour des millions d'enfants déchiquetés ? Se prépare-t-il à peindre des formes neuves? A-t-il dans la tête des histoires qui s'écriront dans une langue que personne n'a encore entendue? La musique de l'esprit surhumain est-elle la forme d'art ultime, le seul langage, celui qui englobe tous les langages? C'est dans le « livre jamais lu » qu'évolue le surhumain. Le " livre jamais lu » doit donc être lu. S'il inspire de la répulsion, il est nécessaire. Nécessaire parce qu'il est humain avant d'être surhumain. Parce qu'il relève la part inhumaine de l'humanité. Parce que l'humanité a besoin de tâter les os de sa propre mort pour entrevoir la vie. Parce que la transcendance avait besoin de l'annihilation de ses pouvoirs pour renaitre de ses cendres. Peut-être. no

* Jean Désy a publié entre autres: Un dernier cadeau pour Cornélia, XYZ, 1989 ; La rêverie du froid, La Liberté, 1991 ; Baie Victor, Septentrion, 1992 ; Voyage au nord du Nord, Le Loup de Gouttière, 1993 ; Docteur Wincot, Le Loup de Gouttière, 1995 ; La saga de Freydis Karlsevni, L'Hexagone, 1995 ; L'espace Montauban, La Liberté, 1996 ; Urgences, Un médecin raconte, La Liberté, 1996; Lettre à ma fille, Le Loup de Gouttière, 1997 ; O Nord, mon amour, Le Loup de Gouttière, 1998 ; Nunavik, carnets de l'Ungava, Les Heures bleues, 2001 ; Le coureur de froid, XYZ, 2001 ; Du fond de ma cabane, Eloge de la foret et du sacré, XYZ, 2002.

\section{"Le Iivre jamais Iu "}

\section{Claire Martin}

signera la prochaine rubrique.

À paraître dans le numéro 91

de Nuit blanche, en librairie le 16 juin 2003
LES NOUVEAUTÉs VARIA
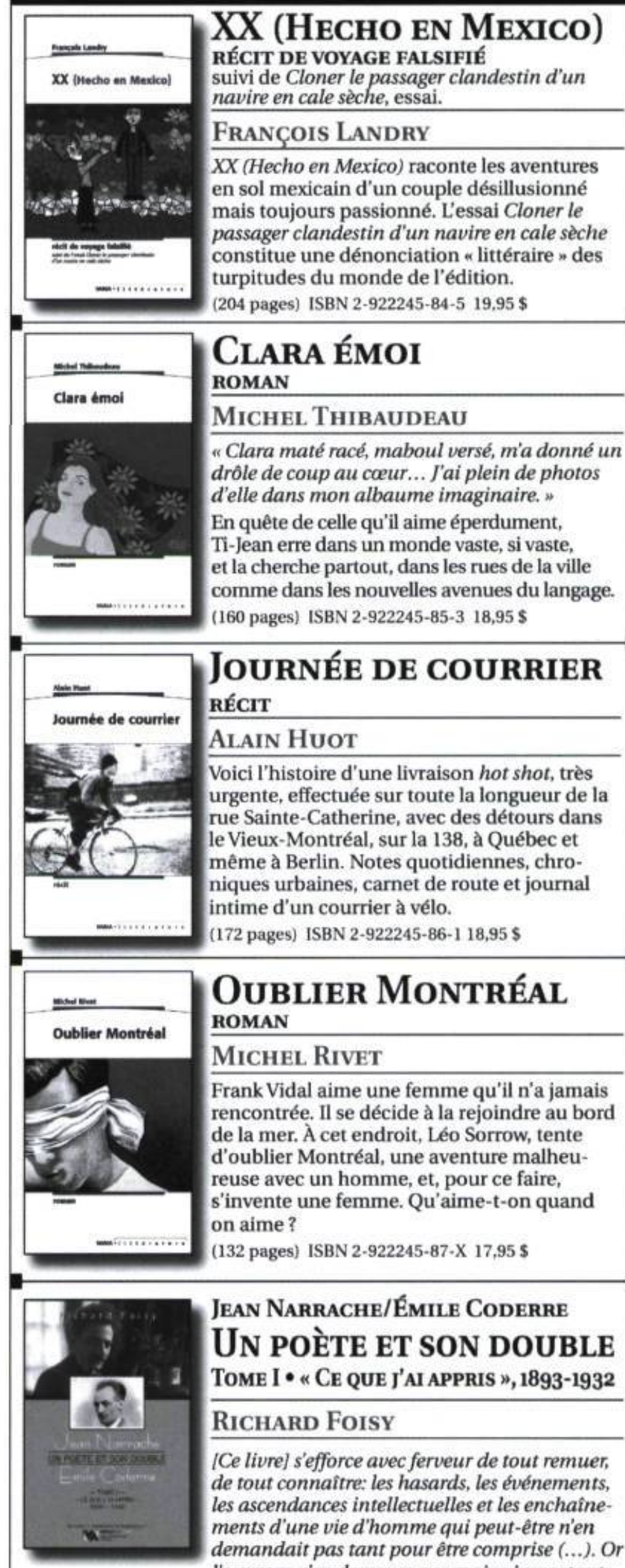

JOURNÉE DE COURRIER RÉCIT

Alain Huot

Voici l'histoire d'une livraison hot shot, très urgente, effectuée sur toute la longueur de la rue Sainte-Catherine, avec des détours dans le Vieux-Montréal, sur la 138, à Québec et même à Berlin. Notes quotidiennes, chroniques urbaines, carnet de route et journal intime d'un courrier à vélo.

(172 pages) ISBN 2-922245-86-1 18,95 \$

\section{Oublier MonTréal} ROMAN

\section{MICHEL RIVET}

Frank Vidal aime une femme qu'il n'a jamais rencontrée. Il se décide à la rejoindre au bord de la mer. À cet endroit, Léo Sorrow, tente d'oublier Montréal, une aventure malheureuse avec un homme, et, pour ce faire, s'invente une femme. Qu'aime-t-on quand on aime?

(132 pages) ISBN 2-922245-87-X 17,95 \$

JeAn NarRache/Émile Coderre UN POÈTE ET SON DOUBLE TOME I • * CE QUE J'AI APPRIS *, 1893-1932

\section{RichaRd FOISY}

[Ce livre] s'efforce avec ferveur de tout remuer, de tout connaitre: les hasards, les événements, les ascendances intellectuelles et les enchainements d'une vie d'homme qui peut-être n'en demandait pas tant pour être comprise (...). Or l'ouvrage n'en demeure pas moins important. Jean-François NADEAU, Le Devoir (510 p. +32 p. de photos) ISBN 2-922245-75-6, 39,95 \$

W W W $\mathbf{W}$ A R I A . C O M 